\title{
Implementation of an Optimization and Simulation-Based Approach for Detecting and Resolving Conflicts at Airports
}

\author{
Paolo Scala $^{1} \quad$ Miguel Mujica Mota $^{2}$ Daniel Delahaye ${ }^{3}$ \\ ${ }^{1,2}$ Aviation Academy, Amsterdam University of Applied Sciences, The Netherlands, \\ \{p.m.scala,m.m.mujica\}@hva.nl \\ ${ }^{3}$ Ecole Nationale de l'Aviation Civile, delahaye@recherche.enac.fr
}

\begin{abstract}
In this paper is presented a methodology that uses simulation together with optimization techniques for a conflict detection and resolution at airports. This approach provides more robust solutions to operative problems, since, optimization allows to come up with optimal or suboptimal solutions, on the other hand, simulation allows to take into account other aspects as stochasticity and interactions inside the system. Both the airport airspace (terminal manoeuvring area), and airside (runway taxiways and terminals), were modelled. In this framework, different restrictions such as speed, separation minima between aircraft, and capacity of airside components were taken into account. The airspace was modeled as a network of links and nodes representing the different routes, while the airside was modeled in a low detail, where runway, taxiways and terminals were modeled as servers with a specific capacity. The objective of this work is to detect and resolve conflicts both in the airspace and in the airside and have a balanced traffic load on the ground.
\end{abstract}

Keywords: optimization, modeling, simulation, airport

\section{Introduction}

Capacity at airports has become a very delicate problem due to the increase of traffic demand and the scarcity of facilities at airports. In Europe it has been seen a growth of traffic of $1.5 \backslash \%$ from 2014 to 2015, and the forecasts say that this growth will continue also for the coming years (Eurocontrol, 2016). Airports are getting busier and busier, especially at the major hubs in Europe, with visible effects as delays occurrences. Looking at the delay from all causes, it can be seen that in the first three months of the year there were between 341\% and 381\% flights delayed on departures, where only delays greater or equal than five minutes are considered (Eurocontrol, 2016). So far, many studies have been conducted in order to alleviate airports from congestion and improve the capacity, some of them focused on the airspace and some other only on the airside. Concerning problems related to the airspace, we can find many works about the sequencing and merging or scheduling of arrivals in the TMA (Beasley et al., 2000; Beasley et al., 2001; Hu and Chen, 2005; Michelin et al., 2009; Balakrishnan and Chandran, 2010; Zuniga et al. 2011). On the other hand we can also find studies related to ground issues such as gate assignment problem (Bolat, 2000; Dorndorf et al., 2007; Kim and Feron, 2012; Narciso and Piera, 2015), scheduling of departures (Pujet et al., 1999; Rathinam et al. 2009; Sandberg et al., 2014; Simaiakis and Balakrishnan, 2015) or airport surface management (Montoya et al., 2010; Simaiakis et al., 2014; Khadilkar and Balakrishnan, 2014). In order to conduct a more precise analysis and obtaining an integrated view of the system, it is better to consider airspace and airside together, so that we can also consider the interactions between the two environments.

The contribution of this paper is that it considers the airport from a holistic perspective, including most of the factors that link airspace and airside and affect the overall airport capacity. Furthermore, another contributions of this work is the employment of an approach that uses optimization together simulation, with the objective of obtaining more robust solutions. On one hand, optimization allows to come up with optimal or suboptimal solutions and, on the other hand, simulation allows to take into account other aspects as stochasticity and interactions inside the system. In literature we can find similar works that employ optimization together with simulation techniques, like in (Mujica, 2015) where an evolutionary algorithm together with discrete event simulation was proposed for the improvement of the check-in allocation, or (Arias et al., 2013), where was presented a model for solving the stochastic aircraft recovery problem employing constraint programming together with simulation.

In this paper a methodology for detecting and resolving conflict at airports is presented, it considers the airport from a holistic view, taking into account both airspace and airside components together. In the methodology presented in this paper an optimal or suboptimal solution is found applying a sliding window approach (Hu and Chen, 2005; Zhan et al., 2010; Furini et al., 2015) together with a meta-heuristic (simulated annealing) (Kirpatrick et al., 1983), after that, the solution provided from the optimization model is tested and validated with the use of a discrete event simulation 
model. Using simulation, it is possible to take into account the stochasticity of the system and the interactions between the entities in the system.

The paper is organized with the following structure, in Section 2 the methodology is explained, in Section 3 a scenario is tested, providing preliminary results and in Section 4 some conclusions are drawn and next steps for this research are delineated.

\section{Methodology}

The methodology presented in this work is constituted by three main steps (Figure 1). The first step aims at modeling the airport taking into account both airspace and airside. In this case the airside was modeled in a "macro" level, where runway, taxiway and terminals were modeled as servers with a specific capacity. The second step consisted in the implementation of an optimization model to obtain a solution for the conflict detection and resolution problem. Finally, in the third step, the solution provided by the optimization model is evaluated by the means of a simulation model in order to test the effectiveness and feasibility of the solution.

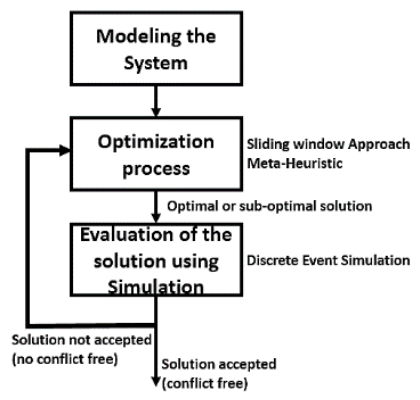

Figure 1. Methodology steps.

\subsection{Airport Modeling}

One of the main contribution of this work is that it considers both airspace and airside of the airport. Concerning the airspace, landing routes in the TMA were modeled, and separation minima between aircraft as well as speed restrictions were included. Regarding the airside, since the objective did not require a detailed evaluation, runway, taxiway and terminals components, were modeled as servers with a specific capacity. First, it is fair to explain the concept behind airspace and airside conflicts. In this framework, it was assumed that any violation of separation minima between aircraft along the airspace routes and at the merging point was considered as a conflict. Values about separation minima are in accordance with the ICAO standards for separation minima due wake vortex turbulence, they are based on the aircraft type which could be light, medium or heavy (see Table 1). Concerning the airside, conflicts were detected when the capacity of runway, taxiway and terminal was exceeded. It is clear that, the objective of detecting and resolving conflict in the airspace and in the airside lead to have a smooth flow of aircraft in the airspace and a balanced load on the airside, which is the main scope of this work. In this work, the case of Paris Charles de Gaulle Airport was considered. Regarding the airspace, standard approach routes (STAR) and final approach segment were modeled. In total there are four different routes coming from different entry points, all of them merge at the merging point before the final approach segment. In Figure 2 the airspace routes taken into account in the model are shown.

Table 1. ICAO wake vortex turbulence separation minima.

\begin{tabular}{|c|c|c|c|c|}
\hline \multicolumn{2}{|c|}{} & \multicolumn{3}{|c|}{ Leading Aircraft } \\
\cline { 3 - 5 } \multicolumn{2}{|c|}{} & Heavy & Medium & Light \\
\hline \multirow{3}{*}{$\begin{array}{c}\text { Trailing } \\
\text { Aircraft }\end{array}$} & Heavy & 4 & 3 & 3 \\
\cline { 2 - 5 } & Medium & 5 & 4 & 3 \\
\cline { 2 - 5 } & Light & 6 & 4 & 3 \\
\hline
\end{tabular}

As a preliminary test, in the model there were considered only one of the three terminal and only two runway (one landing and one departing) of the four runways (two landings and two departing) that constitute the airport airside. Concerning the values chosen for the capacity of the airside components, it is intuitive that runways have the value of capacity equal to one since only one aircraft is allowed to cross the runway at a time. Of the three terminal, terminal 2 was chosen for being tested, due to the availability of data concerning inbound and outbound flight to and from this terminal. Terminal 2 is the biggest of the three terminals in Paris CdG airport, it accommodates all the flights of Air France and SkyTeam members. In Table 2 all the characteristics concerning airside components are listed with their respective values.

Table 2. Characteristics of airside components.

\begin{tabular}{|c|c|}
\hline Airside Component & Capacity \\
\hline Runway 26 R/L & 1 \\
\hline Taxiway Network & 20 \\
\hline Terminal 2 & $176(152$ considered $)$ \\
\hline
\end{tabular}

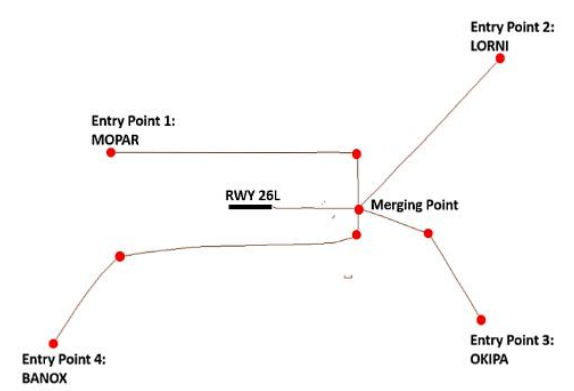

Figure 2. STAR and final approach segment for Rwy $26 \mathrm{~L}$ 


\subsection{Optimization Model}

The optimization model proposed to solve the conflict detection and resolution problem is based on (Ma et al., 2015), where a sliding window approach ( $\mathrm{Hu}$ and Chen, 2005; Zhan et al., 2010; Furini et al., 2015) is used together with a meta-heuristic (simulated annealing) (Kirkpatrick et al., 1983) to solve conflicts in the airspace. The sliding window approach allows to consider an extended time horizon in smaller time frames, dividing the overall problem in sub-problems of smaller size, therefore, decreasing the computational time. Moreover, it allows to treat the problem in a dynamical way, where decisions that are made in each window will affect the decisions to be made in the successive window. The main parameters of the sliding window approach are the size of the window and the size of the shift.

The meta-heuristic used is the simulated annealing (Kirkpatrick et al., 1983), this heuristic is a local search algorithm which is able to escape from local optimum by allowing hill-climb moves in order to find a global optimum.

The main aspect that differentiates this work from the other aforementioned works is that, beside the airspace, airside operations were also included. The new objective becomes the detection and resolution of conflicts both in the airspace and in the airside. The objective of this optimization model is twofold, first it aims at detecting and resolving conflicts in the airspace and also capacity conflicts in the airside, and second is to ensure a smooth flow of aircraft in the airspace and a balanced load on the ground. The decision variables for the problem are: entry time change, entry speed change and pushback time change. The first is the time when aircraft enter the airspace route, the second is the speed that aircraft have when they enter the airspace route and the third is the delay allowed to the aircraft, that are parked at the gate, before they leave the gate and reach the runway for taking off. In Table 3 are shown the values that the decision variables can assume.

Table 3. Value range of the decision variables.

\begin{tabular}{|c|c|}
\hline Decision Variable & Value \\
\hline Entry Time & Between -5 and +30 min \\
\hline Entry Speed & Between -10 and +10 \\
\hline Pushback Time & Between 0 and 5 min \\
\hline
\end{tabular}

In this context, conflicts in the airspace are detected in the following way: node and link detection. Routes are modeled as a network made by nodes and links, in every node and in every link aircraft are tracked by their "time in" and "time out". If the time interval between "time in" and "time out" overlaps for two or more aircraft then a conflict is detected, the same principle is applied for nodes and links. In Table 4 are described the four routes of the airspace plus the final approach route.
Table 4. Characteristics of the routes modeled in the optimization model.

\begin{tabular}{|c|c|}
\hline STAR (Entry point) & $\begin{array}{c}\text { Number of } \\
\text { nodes (links) }\end{array}$ \\
\hline STAR1 (MOPAR) & $4(4)$ \\
\hline STAR2 (LORNI) & $2(2)$ \\
\hline STAR3 (OXIPA) & $2(2)$ \\
\hline STAR4 (BANOX) & $4(4)$ \\
\hline $\begin{array}{c}\text { Final Approach segment (Merging } \\
\text { point) }\end{array}$ & $3(3)$ \\
\hline
\end{tabular}

Concerning airside components as runways, taxiway network and terminal, there were made some assumption about runway occupancy time (for landings and take offs), taxiway occupancy time and turnaround time, they were based on fixed values, in Table 5 these values are listed.

Table 5. Times for airside components in the optimization model.

\begin{tabular}{|c|c|}
\hline $\begin{array}{c}\text { Airside } \\
\text { component }\end{array}$ & Time \\
\hline Runway & $\begin{array}{c}60 \mathrm{sec} \text { landing }-25(\mathrm{H})-30(\mathrm{M})-35(\mathrm{~L}) \text { sec take } \\
\text { off }\end{array}$ \\
\hline Taxiway & $10 \mathrm{~min}$ \\
\hline Terminal & OffBlockTime-InBlockTime \\
\hline
\end{tabular}

\subsection{Simulation Model}

The simulation model is built using a discrete event simulation approach. The employment of this approach allows to take into account the stochasticity of the system and also the interactions inside the system (Banks et al., 2010). For example, values related to runway occupancy time, taxiway occupancy time and turnaround time were modeled following probability distributions, whereas, in the optimization model these values were assumed as deterministic. Another factor that differentiate the simulation approach from the optimization one is the speed profile. In the optimization model the acceleration used is fixed and the time in and time out for each node and link is calculated in a static way based on the length of the link, whereas in the simulation model, speed is regulated using a fixed acceleration that is updated each second. In this way, the speed profile will be more realistic. Moreover, during the descending approach the simulation model does not allow aircraft to fly below a certain speed threshold, indicated as lower bound speed. In the simulation model, although were modeled the same routes, these routes were modeled using more nodes and links. The theory behind this approach is to construct a network of equidistant nodes in order to detect conflict more accurately along the route. In the model nodes are distanced by 5 NM from each other, which is assumed as an acceptable distance to make sure to do not miss any conflict along the route. Based on that, 
it is likely to find, under the same conditions, more conflicts in the simulation model than in the optimization model. Due to this network structure, in the simulation model conflicts are detected only on the nodes and not on links. Concerning the detection of conflicts, it is applied the same principle as in the optimization model. In Table 6 the main characteristics of the airspace network are listed.

Table 6. Route network in the simulation model.

\begin{tabular}{|c|c|}
\hline STAR (Entry point) & $\begin{array}{c}\text { Number of } \\
\text { nodes (links) }\end{array}$ \\
\hline STAR1 (MOPAR) & $11(11)$ \\
\hline STAR2 (LORNI) & $7(7)$ \\
\hline STAR3 (OXIPA) & $7(7)$ \\
\hline STAR4 (BANOX) & $15(15)$ \\
\hline $\begin{array}{c}\text { Final Approach segment (Merging } \\
\text { point) }\end{array}$ & $3(3)$ \\
\hline
\end{tabular}

The main objective of the simulation model is to test if the solution that comes from the optimization model is feasible also in a more accurate scenario.

Regarding the detection of conflicts on the airside, in the simulation are used the same principles as in the optimization model, with the only difference that times are based on probability distributions and therefore also variability is included, instead, in the optimization model times were based on fixed values, making it more predictable and static.

\subsubsection{Validation of the Simulation Model}

In order to validate the model, we have conducted a cross validation (Geisser, 1975). This type of validation consists in dividing the set of data in two parts, called set training set and testing respectively, and then using the training set for calibrating the simulation parameter in order to get an outcome that resembles the trailing set data sample. After that, the testing set and the simulation outcome are compared in order to see if the simulation is a good predictive of the data. In this case it was used the first half of the input flight schedule as training set and the second half of the input flight schedule as testing set. Figures 3 and 4 show the daily trend of traffic of the testing set and the simulation outcome after the calibration of the data. In Table 7 are shown the parameters that were used in the simulation model. Figures 5 and 6 show the outcome from the simulation and the testing set. In both sets, Figures 3 and 4 and Figures 5 and 6 , it can be seen that the hourly traffic from the simulation and the hourly traffic obtained from the real flight schedule follow the same trend. The mean square error estimator was used to estimate the accuracy of the result obtained from the simulation compared to the real data set.

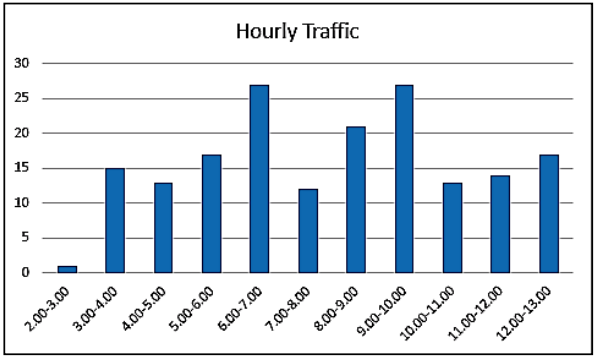

Figure 3. Trend of the daily traffic from the training set.

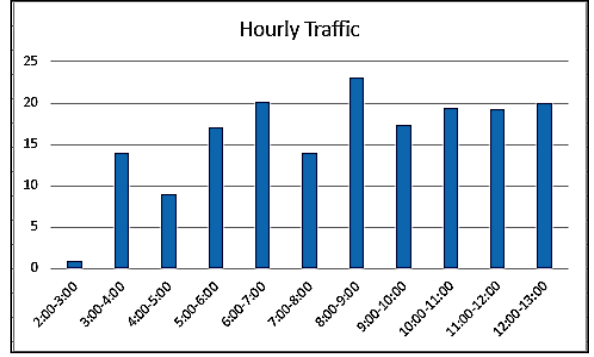

Figure 4. Outcome from the simulation model using the training set as data sample.

In Tables 8 and 9 the values of mean square errors for each hour of traffic are listed, for the training set and testing set, respectively. By observing these values it can be noticed that the ones related to the testing set are not that high which means that the simulation is relatively reliable in predicting the system.

Table 7. Parameters of the airside components in the simulation model.

\begin{tabular}{|c|c|}
\hline Airside component & Time \\
\hline Runway & Triangular $(0.5,0.75,1) \mathrm{min}$ \\
\hline Taxiway network & Triangular $(8,10,12) \mathrm{min}$ \\
\hline Terminal & Triangular $(25,35,45) \mathrm{min}$ \\
\hline
\end{tabular}

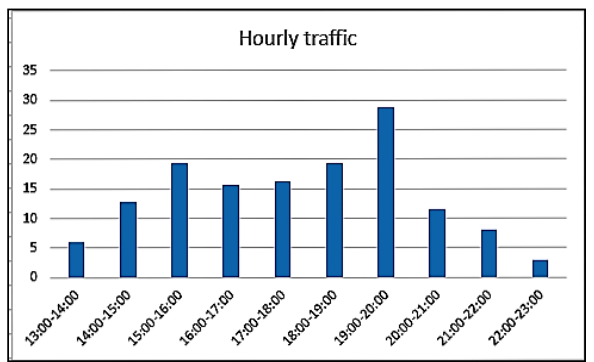

Figure 5. Outcome from the simulation model for the second half of the data sample (testing set).

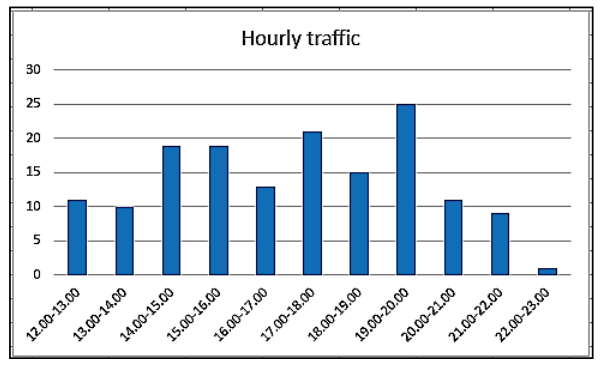

Figure 6. Trend of the daily traffic from the testing set. 
Table 8. Mean square error values for training set.

\begin{tabular}{|c|c|}
\hline Hour & mean square error \\
\hline $2: 00-3: 00$ & 0 \\
\hline $3: 00-4: 00$ & 1 \\
\hline $4: 00-5: 00$ & 15.76 \\
\hline $5: 00-6: 00$ & 0.2 \\
\hline $6: 00-7: 00$ & 47.16 \\
\hline $7: 00-8: 00$ & 8.73 \\
\hline $8: 00-9: 00$ & 93.76 \\
\hline $9: 00-10: 00$ & 43 \\
\hline $10: 00-11: 00$ & 29.2 \\
\hline $11: 00-12: 00$ & 10.53 \\
\hline $12: 00-13: 00$ & 9 \\
\hline
\end{tabular}

Table 9. Mean square error values for testing set.

\begin{tabular}{|c|c|}
\hline Hour & mean square error \\
\hline $13: 00-14: 00$ & 16 \\
\hline $14: 00-15: 00$ & 38.6 \\
\hline $15: 00-16: 00$ & 0.66 \\
\hline $16: 00-17: 00$ & 7.6 \\
\hline $17: 00-18: 00$ & 23.8 \\
\hline $18: 00-19: 00$ & 20.2 \\
\hline $19: 00-20: 00$ & 16.5 \\
\hline $20: 00-21: 00$ & 0.7 \\
\hline $21: 00-22: 00$ & 9.3 \\
\hline $22: 00-23: 00$ & 4 \\
\hline
\end{tabular}

\section{Scenario and Results}

In order to test the goodness of the methodology there have been conducted a series of preliminary tests primarily to tune the parameters of the optimization model in the specific the parameters of the sliding window approach and of the simulated annealing metaheuristic. Values related to the sliding window parameters are listed in Table 10.

Table 10. Sliding window parameters.

\begin{tabular}{|c|c|}
\hline Parameter & Value \\
\hline Window duration & $2 \mathrm{Hrs}$ \\
\hline Shift & $30 \mathrm{~min}$ \\
\hline
\end{tabular}

Concerning the experiments, one scenario was tested based on a flight schedule related to a specific day. Once the optimized solution is ready it will be tested with the use of the simulation model developed and discussed in Section 2.3.

\subsection{Scenario}

In the flight schedule that was used, three different typology of flights were identified: arrivals, departures and arrivals and departures. The first type means that aircraft will arrive and stay at the gate for the whole day without departing again to another destination, in the flight schedule you can find most of them during the middle of the day and in the evening. The second type, is departure flight which means that those aircraft are already parked in one of the gate and they will depart to a destination, usually you can find those type of flights during the morning. Finally, the third type, arrivals and departures, are those flights that arrive from an origin, they park at the gate and then they depart to another destination, these flights represent the majority of the flights in the schedule. In Table 11 the structure of the flight schedule, according to the flight type, is showed.

Table 11. Flight schedule structure for the scenario.

\begin{tabular}{|c|c|}
\hline \multicolumn{2}{|c|}{ Scenario } \\
\hline Arrivals & 149 \\
\hline Departures & 48 \\
\hline Arrivals and departures & 91 \\
\hline
\end{tabular}

As it can be seen from the figures above, in the first schedule there are 3 peaks: 7.00-8.00, 10.00-11.00 and 20.00-21.00, with 31, 24 and 25 air traffic movements, respectively.

\subsection{Results from the Optimization Model}

Table 12 summarize the results obtained running the optimization model before and after the implementation of the simulated annealing meta-heuristic. Looking at the table above, it can be noticed that the optimization model is able to reach a conflict free situation in 120 sec., when the initial solution without optimization registered in total 307 conflicts, 121 on nodes, 144 on links and 42 on the runway. Moreover, it can be noticed that taxiway and terminals are not affected by conflicts both before and after the optimization process, proving that under the given traffic, the capacity of the two components is able to handle this traffic without incurring in congestion problems.

Table 12. Results before and after optimization process.

\begin{tabular}{|c|c|c|}
\hline & before opt & after opt \\
\hline $\begin{array}{c}\text { Computational } \\
\text { time }\end{array}$ & $101.883 \mathrm{sec}$ & $120.18 \mathrm{sec}$ \\
\hline Total objective & 307 & 0 \\
\hline Node conflicts & 121 & 0 \\
\hline Link conflicts & 144 & 0 \\
\hline Runway conflicts & 42 & 0 \\
\hline Taxiway conflicts & 0 & 0 \\
\hline Terminal conflicts & 0 & 0 \\
\hline
\end{tabular}

\subsection{Results from the Simulation Model}

After running the optimization model and obtaining an optimal solution, this solution has been tested by means 
of a discrete event simulation model. It was simulated the whole day and there were run 30 replication. Tables 13 and 14 show the results obtained by the simulation model. For simplicity, we have named as the conflict detected on the nodes in the airspace routes were named "airspace conflicts". Moreover, in order to have a better idea of how many aircraft are involved in the conflicts, the number of aircraft affected by at least one conflict in the airspace were collected.

Table 13. Results from simulation model before the optimization process.

\begin{tabular}{|c|c|c|c|c|}
\hline & Min & Avg & Max & St. dev \\
\hline Aircraft conflicts & 57 & 57 & 57 & 0 \\
\hline Airspace conflicts & 256 & 256 & 256 & 0 \\
\hline Runway In conflicts & 86 & 92 & 98 & 1.139 \\
\hline Runway Out conflicts & 1 & 1.55 & 3 & 0.4255 \\
\hline Taxiway conflict & 0 & 0 & 0 & 0 \\
\hline Terminal conflicts & 0 & 0 & 0 & 0 \\
\hline
\end{tabular}

Table 14. Results from simulation model after the optimization process.

\begin{tabular}{|c|c|c|c|c|}
\hline & Min & Avg & Max & St. dev \\
\hline Aircraft conflicts & 38 & 38 & 38 & 0 \\
\hline Airspace conflicts & 180 & 180 & 180 & 0 \\
\hline Runway In conflicts & 49 & 51.3 & 55 & 0.7385 \\
\hline Runway Out conflicts & 1 & 1.41 & 2 & 0.3272 \\
\hline Taxiway conflict & 0 & 0 & 0 & 0 \\
\hline Terminal conflicts & 0 & 0 & 0 & 0 \\
\hline
\end{tabular}

In the simulation model it is likely to find more conflicts in the airspace than in the optimization model, due to its different route structure that provides more nodes and links than the route structure of the optimization model. Another distinction was made between "runway in" and "runway out", which refers to runway used for landings and runway used for departures, respectively. Looking at the results, we can see that there are still conflicts, they are mainly concentrated in the airspace were we have 180 conflicts on nodes occurred to 38 aircraft. No source of variability affect these results because there is not any source of stochasticity in the values related to the airspace. We have in average 51.53 conflicts for runway in and 1.41 conflicts for runway out, while no conflicts are detected for taxiway and terminal. It is noticeable that, even though after the optimization process in the simulation model there are still conflicts, they have decreased sensibly, compared with the scenario without optimization process. It proves that the solution provided by the optimization process is able to reduce the number of conflicts, but it is not enough to achieve a conflict free solution.

\section{Conclusions and Future Work}

In this paper, a methodology for detecting and resolving conflicts at airports is presented. The methodology consists in the implementation of optimization together with discrete event simulation techniques in order to come up with more robust solutions. The optimization model was solved using a sliding window approach and the simulated annealing meta-heuristic. With the use of a discrete event simulation model, the methodology aimed at evaluating the solution given from the optimization model, in a real and more accurate environment. In this work, Paris Charles de Gaulle Airport was taken as a case study and one scenario was tested based on the flight schedule of a specific day.

From the results, we found that the optimization was able to find an optimal (conflict free) solution. When the solution was tested using the simulation model it was found that, although conflicts were sensibly decreased compared to the non optimized scenario, there were still a lot of conflicts both in the airspace and on the runway. From this results it is possible to conclude that the solution from the optimization model was not feasible, and therefore, the optimization model needs further refinements in order to produce a more robust ad feasible solution.

Next steps for this research are in accordance with the results, therefore, the optimization model needs to be refined in order to be more accurate. Furthermore, the airside can be modeled in a more detail, including taxiway routes and gate assignment.

\section{Acknowledgements}

The authors would like to thank the Aviation Academy of the Amsterdam University of Applied Sciences and Ecole Nationale de l'Aviation Civile for the support to perform this study.

\section{References}

P. Arias, D. Guimarans, G. Boosten, M. Mujica. A methodology combining optimization and simulation for real applications of the Stochastic Aircraft Recovery Problem. In Conference proceedings of the EUROSIM13. Cardiff, U.K, 2013. doi: 10.1109/EUROSIM.2013.55.

H. Balakrishnan, B. Chandran. Algorithms for scheduling runway operations under constrained position shifting. Operation Research, 58(6): 1650-1665, 2010.

J. Banks, J.S. Carson, B. Nelson, D.M. Nicol. Discrete-Even system Simulation. 5th ed. Upper Saddle River, NJ: Pearson. 2010.

J.E. Beasley, M. Krishnamoorthy, Y.M. Sharaiha, D. Abramson. Scheduling aircraft landings: the static case. Transportation Science, 34(2): 180-197, 2000.

J.E. Beasley, J. Sonander, P. Havelock. Scheduling aircraft landings at London heathrow using a population heuristic. Journal of the Operational Research Society, 52(5): 483493, 2001. doi: 10.1057/palgrave.jors.2601129. 
A. Bolat. Procedures for providing robust gate assignments for arriving aircraft. European Journal of Operational Research, 120(1): 63-80, 2000. doi: 10.1016/S03772217(98)00375-0.

U. Dorndorf, A. Drexl, Y. Nikulin, E. Pesch. Flight gate scheduling: State-of-the-art and recent developments. Omega, 35(3): 326-334, $2007 . \quad$ doi: 10.1016/j.omega.2005.07.001.

Eurocontrol, Industry monitor. Issue N180-181-182. 2016.

F. Furini, M.F. Kidd, C.A. Persiani, P. Toth. Improved rolling horizon approaches to the aircraft sequencing problem. Journal of Scheduling, 18(5): 435-447, 2015. doi: 10.1007/s10951-014-0415-8.

S. Geisser. The predictive sample reuse method with aplications. J. Amer. Statist. Ass., 70(350): 315-334, 1975.

X. Hu, W. Chen. Receding Horizon Control for Aircraft Arrival Sequencing and Scheduling. IEEE Transactions On Intelligent Transportation Systems, 6(2): 189-197, 2005. doi: 10.1109/TITS.2005.848365

H. Khadilkar, H. Balakrishnan. Network Congestion Control of Airport Surface Operations. Journal of Guidance, Control, and Dynamics, 37(3): 933-940, 2014.

S.H. Kim, E. Feron. Impact of gate assignment on gateholding departure control strategies. In Conference proceedings of the Digital Avionics Systems Conference (DASC), Williamsburg, (Virginia, USA), 2012. doi: 10.1109/DASC.2012.6382350.

S. Kirkpatrick, C.D. Gelatt, M.P. Vecchi. Optimization by Simulated Annealing. Science, 220(4598): 671-680, 1983. doi: $10.1126 /$ science.220.4598.671.

J. Ma. Aircraft merging and sequencing problems in TMA. Master Thesis, Enac, 2015.

A. Michelin, M. Idan, J.L. Speyer. Merging of air traffic flows. In Conference proceedings of the AIAA Guidance, Navigation, and Control Conference. Chicago, Illinois, 1013 August 2009.

J. Montoya, Z. Woord, S. Rathinam, W. Malik. A Mixed Integer Linear Program for Solving a Multiple Route Taxi Scheduling Problem. In Conference proceedings of the AIAA Guidance, Navigation, and Control Conference. pages 1-15, Toronto, (Ontario, Canada), August 2-5 2010.

M. Mujica. Check-in allocation improvements through the use of a simulation-optimization approach. Transportation Research Part A, 77: 320-335, 2015. doi: 10.1016/j.tra.2015.04.016.

M.E. Narciso, M.A. Piera. Robust gate assignment procedures from an airport management perspective. Omega. 50: 8295, 2015. doi: 10.1016/j.omega.2014.06.003.

N. Pujet, B. Declaire, E. Feron. Input-output modeling and control of the departure process of congested airports. In Conference proceedings of the Guidance, Navigation, and Control Conference and Exhibit, pages 1835-1852, Portland, (Oregon, USA), August 9-11 1999. doi: 10.2514/6.1999-4299.

S. Rathinam, Z. Wood, B. Sridhar, Y. Jung. A Generalized Dynamic Programming Approach for a Departure Scheduling Problem. In Conference proceedings of the AIAA Guidance, Navigation, and Control Conference, pages 1-12, Chicago, (Illinois, USA), August 10-13 2009.
M. Sandberg, I. Simaiakis, H. Balakrishnan, T.G. Reynolds, R.J. Hansman. A Decision Support Tool for the Pushback Rate Control of Airport Departures. IEEE Transactions on Human-Machine Systems, 44(3): 416-421, 2014. doi: 10.1109/THMS.2014.2305906.

I. Simaiakis, H. Khadilkar, H. Balakrishnan, T.G. Reynolds, R.J. Hansman. Demonstration of reduced airport congestion through pushback rate control. Transportation Research Part A: Policy and Practice, 66: 251-267, 2014. doi: 10.1016/j.tra.2014.05.014.

I. Simaiakis, H. Balakrishnan. A Queuing Model of the Airport Departure Process. Transportation Science, 50(1): 94-109, 2015.

Z. Zhan, J. Zhang, Y. Li, O. Liu, S.K. Kwok, W.H. Ip, O. Kaynak. An Efficient Ant Colony System Based on Receding Horizon Control for the Aircraft Arrival Sequencing and Scheduling Problem. IEEE Transactions on Intelligent Transportation System, 11(2): 399-412, 2010. doi: 10.1109/TITS.2010.2044793.

C.A. Zuniga, D. Delahaye, M.A. Piera. Integrating and Sequencing Flows in Terminal Maneuvering Area by Evolutionary Algorithms. In Conference proceedings of the DASC 2011, 30th IEEE/AIAA Digital Avionics Systems Conference, Seattle, United States, 2011. doi: 10.1109/DASC.2011.6095980.

C.A. Zuniga, M.A. Piera, S. Ruiz, I. Del Pozo. A CD $\&$ CR causal model based on path shortening/path stretching techniques. Transportation Research Part C, 33: 238-256, 2013. 